\title{
EMPIRICAL ADEQUACY AND RAMSIFICATION, II
}

JEFFREY KETLAND

University of Edinburgh

\section{INTRODUCTION}

\subsection{Ramsey Sentences}

This paper returns to the topic of Ketland 2004, which aimed to clarify the Newman objection to scientific structuralism. Several ways of formulating the propositional knowledge claims associated with scientific structuralism involve the appearance of a Ramsey sentence, in one form or another. Some structuralists "ramsify" the linguistic formulations of theories explicitly (e.g., Zahar 2001, Appendix), while others "ramsify" in a less obvious manner (e.g., the content of any "structural representation claim" seems to be a Ramsey sentence). However, there is a problem. In a 1928 review of Russell's structuralism, M.H.A. Newman pointed out that these "structural representation claims" reduce to cardinality claims. In 1985, Demopoulos and Friedman applied this criticism to argue that the truth of a theory's Ramsey sentence is equivalent to the theory's empirical adequacy plus a condition on the cardinality of the world. In particular, the "structural content" of a theory reduces to cardinality content. In Ketland 2004, the main result obtained was that a Ramsey sentence $\Re(\Theta)$ is true just if the theory $\Theta$ has an empirically correct full model with sufficiently many objects. Thus, not much more than empirical adequacy is required for $\mathfrak{R}(\Theta)$ to be true: so, structuralism collapses to anti-realism (cf., van Fraassen's constructive empiricism).

The notion of a Ramsey sentence first appears in Frank Ramsey's "Theories" (1929), discussing the role of theoretical predicates (observational predicates are supposed, via a "dictionary" or "correspondence rules", to "interpret" the theoretical predicates). Consider a statement of the form $\Theta\left(\mathbf{T}_{1}, \ldots, \mathbf{T}_{n}\right)$, with predicates $\mathbf{T}_{1}, \ldots, \mathbf{T}_{n}$ occurring in $\Theta$. The corresponding Ramsey sentence has the form:

(1) There are relations $R_{1}, \ldots, R_{n}$ such that $\Theta\left(R_{1}, \ldots, R_{n}\right)$. 
Syntactically, the Ramsey sentence replaces the predicates $\mathbf{T}_{i}$ with secondorder variables $R_{i}$, and then prefixes the result with existential quantifiers for those variables. We say that the $\mathbf{T}_{i}$ have been "ramsified". ${ }^{1}$ In effect, (1) expresses that the condition $\Theta$ is satisfiable. More generally, a Ramsey sentence has the form:

(2) There are relations $R_{1}, \ldots, R_{n}$ on domain $D$ such that $\Theta\left(D, R_{1}, \ldots, R_{n}\right)$.

Example 1. Consider Max Born. The following is true:

(3) Max Born is the grandfather of Olivia Newton John.

Applying second-order $\exists$-I to the predicate "grandfather", (3) implies:

(4) There is a relation $R$ such that Max Born bears $R$ to Olivia Newton John.

This is the Ramsey sentence of (3). Call it $\Re(3)$. There is a sense in which the truth of $\Re(3)$ is nearly trivial. More exactly,

(5) $\Re(3)$ is true iff (3) is satisfiable on a domain containing Max and Olivia.

Example 2: Suppose $L$ is a formalized language and $\Theta$ is an $L$-theory. Consider the claim,

(6) The theory $\Theta$ has a model with domain $D$.

This is equivalent to,

(7) There are relations $R_{1}, \ldots, R_{n}$ on $D$ such that $\left(D, R_{1}, \ldots, R_{n}\right) \vDash \Theta$.

\footnotetext{
1 "Ramsified" or "Ramseyfied"? As I understand the terminological use, the phrase "ramsified" is an allusion to Ramsey's discussion of Russell's ramified theory of types. Ramsey noted that the ramified theory of types is an unnecessary complication over the simple theory. And this observation has been expressed as a joke: "type theory needs ramsification, not ramification".
} 
Thus, " $\Theta$ has a model" is, in effect, a Ramsey sentence.

Example 3. Suppose that $\mathbf{M}$ is a mathematical structure, as might crop up in the mathematical development of a scientific theory. For example, M might be the abstract 4-dimensional Minkowski spacetime, $\left(\mathbf{R}^{4}, \eta_{a b}\right)$. Consider claims of the form:

\section{Structural Representation Claims}

(8a) M "represents" the world.

(8b) $\mathbf{M}$ is "isomorphic" to the world.

(8c) The world "exemplifies" $\mathbf{M}$.

(8d) The world "instantiates" $\mathbf{M}$.

It's unclear what this kind of claim means. One analysis of $(8 a)-(8 d)$ seems to be the following:

(9) There are $R_{1}, \ldots, R_{n}$ on $D \subseteq$ the world, with $\mathbf{M} \cong\left(D, R_{1}, \ldots, R_{n}\right)$.

So, (8a)-(8d) are, in effect, Ramsey sentences. Under this analysis, structural representation claims are Ramsey sentences.

Example 4. Suppose that Tim is the set of temporal instants and Bef is the physical relation of time-ordering expressed, in English, by " $t_{1}$ is before $t_{2}$ ". Then a simple theory of time, $\Theta_{\mathrm{T}}$, may be expressed by the statement:

(10) $($ Tim, Bef $) \cong\left(\mathbf{R},<_{\mathbf{R}}\right)$.

One may "ramsify" $\Theta_{\mathrm{T}}$ as follows:

(11) There is a set $X \subseteq$ the world and a relation $S$ on $X$ with $(X, S) \cong$ $\left(\mathbf{R},<_{\mathbf{R}}\right)$.

Example 5. Maxwell's equations for electromagnetism may be written as follows:

(12) For any spacetime point $p,(\nabla \cdot \mathbf{B})(p)=0$, and $(\nabla \cdot \mathbf{E})(p)=\rho(p)$, etc.

This is "ramsified" as follows: 
(13) There exist vector fields $\mathbf{X}, \mathbf{Y}, \mathbf{Z}$ and a scalar field $F$, on spacetime such that, for any spacetime point $p,(\nabla \cdot \mathbf{X})(p)=0$, and $(\nabla \cdot \mathbf{Y})(p)=F(p)$, etc.

\subsection{Structuralism}

Throughout the $20^{\text {th }}$ century, versions of scientific structuralism have been proposed by many philosophers of science, including Russell, Carnap, (G.) Maxwell, Sneed, Zahar, Worrall, Stegmüller, Redhead, French, Ladyman and others. ${ }^{2}$ Simplifying somewhat, one school of scientific structuralism (Maxwell 1970, Worrall 1989, Zahar 2001, et al.) advocates an epistemological view, embodied by a slogan along the lines of "all we can know of the external world is its structure". Zahar and Worrall relate their arguments back to Poincaré, and further back to Kant. These views are usually motivated by epistemological considerations about what we can come to know about the world. For Poincaré, Zahar and Worrall, these considerations involve theory change in science.

It is probably sensible to begin with the version of epistemological structuralism given by Russell (Russell 1919, 1927), who wished to analyse the relation of cognition to the external world. In modern terminology, Russell's idea is that a "phenomenal structure" of some sort is built up in the mind on the basis of perceptual experience. This contains the percepts arranged in a relational structure. The mind is directly acquainted with this structure. But we are, on Russell's view, not directly acquainted with external reality. In a sense, we know it only by "description". To cash this out, Russell suggested, again with modern terminology, that the relation of this phenomenal structure to the world is that it is "isomorphic" to the world. For example, let the phenomenal domain $P$ be the set of auditory pitches $\{\mathrm{Do}, \mathrm{Re}, \mathrm{Mi}, \mathrm{Fa}, \mathrm{So}, \mathrm{La}, \mathrm{Ti}\}$, familiar to readers who have seen The Sound of Music. Let the distinguished relation $H$ be the perceptual relation "has higher pitch than". Then the structure $(P, H)$ is the corresponding phenomenal structure. Russell's idea is that experience brings us into direct acquaintance with a phenomenal structure $\mathbf{M}_{\text {phen. Then our scientific }}$ knowledge of the external world, in general, takes the following form:

(14a) The world $W$ exemplifies (or: instantiates) $\mathbf{M}_{\text {phen }}$.

\footnotetext{
${ }^{2}$ In addition, we have other forms of "structuralism", including Saussure in linguistics, Levi-Strauss in anthropology, and the more plausible mathematical structuralism of Shapiro and Resnik.
} 
(14b) $\mathbf{M}_{\text {phen }}$ represents (or: is isomorphic to) the world $W$.

These are "structural representation claims" whose obvious analysis is a Ramsey sentence, thus:

(15) For some $D \subseteq W$, there are $R_{1}, \ldots, R_{n}$ on $D$ with $\left(D, R_{1}, \ldots, R_{n}\right) \cong$ $\mathbf{M}_{\text {phen. }}$.

There is a connection between Russell's structuralism and a more recent position concerning the nature of theories, the "model-theoretic" conception of theories, which holds that a scientific theory is, or is to be "presented" as, a "class of mathematical structures" or a "class of models". The main problem with the model-theoretic conception of theories concerns its repudiation of semantics. ${ }^{3}$ For, if a theory is a class of mathematical structures, it is extremely unclear what it means to say of a theory (a collection of structures) that it is true. There is no familiar sense in which structures are truth-bearers. In contrast, under ordinary Tarskian semantics, truth bearers are formulas of some interpreted language $\mathbf{L}$. An interpreted language $\mathbf{L}$ may be construed as a pair $(L, \mathbf{M})$, where $L$ is an uninterpreted language, and $\mathbf{M}$ is an $L$-structure (or interpretation). Then truth of a formula $\varphi$ in $\mathbf{L}$ is precisely defined in the usual way. I.e., $\varphi$ is true in $\mathbf{L}$ iff $\mathbf{M}$ $\vDash \varphi$. Alternatively, truth bearers may be taken to be propositions (e.g., those expressed by sentences in an interpreted language). Indeed, in my view, scientific theories simply are collections of propositions. But models and structures are not truth-bearers. ${ }^{4}$ So, what does it mean to say of a structure (or model) that it is "true"? For example, given a class $\Sigma$ of structures (e.g., the models of Peano arithmetic; the countable dense linear orderings without endpoints, etc.), what could the following mean:

(16) The class $\Sigma$ of structures is true?

Perhaps, (16) means something like:

\footnotetext{
${ }^{3}$ Ironically, the model-theoretic conception is sometimes called the "semantic view" of theories. But the central concepts of semantics are truth, meaning and reference. And there is no defined notion of a structure, or model, being "true".

${ }^{4}$ One can, in fact, define an auxiliary notion of an "interpretation" $\mathfrak{I}$ of a structure $\mathbf{M}$. One can go on to define "M is correct, under $\mathfrak{I}$ ". But we lack the space here to discuss this.
} 
(17) Some $\mathbf{M} \in \Sigma$ "represents" (or "is isomorphic to") the world.

This is a "structural representation claim" again, whose meaning is, presumably:

(18) For some $\mathbf{M} \in \Sigma$, there are $R_{1}, \ldots, R_{n}$ on $D \subseteq$ the world such that $\mathbf{M} \cong\left(D, R_{1}, \ldots, R_{n}\right)$.

Again, this is a Ramsey sentence. So, advocates of the model-theoretic conception of theories are committed to ramsification, but in a less obvious way. The model-theoretic conception of scientific theories tends to be associated with a certain subschool of scientific structuralism: "ontic structural realism" or "ontological structuralism" (see Ladyman 1998, French \& Ladyman 2003, Brading \& Landry 2005 et al.). Insofar as I grasp its claims, it appears to rest on two main lines of argument. First, there are certain arguments about indiscernibility in quantum theory and spacetime theory, alleged to have structuralist consequences; second, a preference for the model-theoretic conception of scientific theories. In contrast with the idea that our knowledge is limited to the world's "structure", the slogan is something like "all there is is structure" (Brading \& Landry 2004). This is baffling. The standard conception of structure is the usual one from logic and mathematics: a domain, with some sequence of distinguished relations. So, does "all there is is structure" mean that reality has a domain and a sequence of distinguished relations? Is ontological structuralism the view that:

\section{Reality is a Structure}

(19) Reality itself is a structure, of the form $\left(W, R_{1}, \ldots, R_{n}\right)$ ?

If one makes this metaphysical assumption then the Newman objection discussed below does not apply. For, in that case, claiming that a given structure $\mathbf{M}$ is "isomorphic to the world" is then the claim that $\mathbf{M} \cong$ $\left(W, R_{1}, \ldots, R_{n}\right)$. This is non-trivial. For example, suppose that reality, at bottom, has two basic distinguished 3-place relations, $R_{1}$ and $R_{2}$. Suppose our representing structure $\mathbf{M}$ is the usual field $(\mathbf{R},+, \times)$ of real numbers. Then the claim that $(\mathbf{R},+, \times) \cong\left(W, R_{1}, R_{2}\right)$ is highly non-trivial. However, if one does not make the "reality is a structure" assumption, the Newman objection does apply, and the structural representation claim " $\mathbf{M}$ is isomorphic to the world" is equivalent to "The world has cardinality at least $|\mathbf{M}|$ ". As 
things stand, I do not know whether or not advocates of ontological structuralism do or don't make this metaphysical assumption.

In contrast, the epistemological school of scientific structuralism tends to maintain that theories be identified with their linguistic formulations, holding the "cognitive content" of a theory $\Theta$, expressed in an interpreted language $\mathbf{L}$, to be given by its Ramsey sentence $\Re(\Theta)$ (see, e.g., Carnap 1956). The epistemological claim is that when a theory $\Theta$ satisfies certain conditions of epistemic warrant (predictive, explanatory success, etc.), one is justified in accepting that $\mathfrak{R}(\Theta)$ is true, or approximately true. This is meant to implement the vague idea that all we know of reality is its "structure", while its "nature" remains forever hidden. ${ }^{5}$

\subsection{Newman's Objection}

A central problem with structuralism, epistemological or ontological, is the objection given by the Cambridge mathematician M.H.A Newman in a short critical review (Newman 1928) of Russell's Analysis of Matter (Russell 1927). After quoting some examples of Russellian structural representation claims, Newman comments,

[A]11 we can say is, "There is a relation $R$ such that the structure of the external world with reference to $R$ is M". Now I have already pointed out that such a statement expresses only a trivial property of the world. Any collection of things can be organised so as to have the structure $\mathbf{M}$, provided there are the right number of them. Hence the doctrine that only structure is known involves the doctrine that nothing can be known that is not logically deducible from the mere fact of existence, except ("theoretically") the number of constituting objects. (Newman 1928, p.144; slight change in notation.)

So, if all that we know of reality is that it "instantiates" a structure $\mathbf{M}$, then it follows that we know nothing more than a lower bound on the cardinality of reality. The mathematical point is this:

\footnotetext{
${ }^{5}$ Ramsey sentences crop up elsewhere, including Lewis's 1970 view concerning how to "define" theoretical terms, and versions of functionalism in philosophy of mind. (A Lewis sentence is stronger than a Ramsey sentence, as it contains uniqueness quantifiers.)
} 


\section{Basic Mathematical Point}

The following statements are equivalent:

(a) There exists a structure $\mathbf{M}_{W}$ on some subset of $W$ such that $\mathbf{M}_{W} \cong \mathbf{M}$.

(b) $|\mathbf{M}| \leq|W|$.

Consider again the structural representation claims of the sort associated with the model-theoretic conception of theories:

(20a) Minkowski spacetime $\left(\mathbf{R}^{4}, \eta_{a b}\right)$ "represents" (or is "isomorphic" to) the world.

(20b) The world instantiates, or exemplifies, Minkowski spacetime $\left(\mathbf{R}^{4}, \eta_{a b}\right)$.

Newman's objection is that these are equivalent to saying:

(21) The cardinality of the world is at least $2^{\mathrm{N}_{0}}$.

Thus, whether a class $\Sigma$ of structures is "true" or not depends merely on the cardinality of the world.

For epistemological structuralism, the situation is more complicated. Consider a scientific theory $\Theta$, expressed in an interpreted language $\mathbf{L}$ with domain $W$. Suppose first that all predicates are "ramsified". Then Newman's point tells us that,

(22) $\mathfrak{R}(\Theta)$ is true iff $\Theta$ has a full model with cardinality $|W|$.

So, the truth of $\Re(\Theta)$ reduces to a satisfiability condition, weaker than $\Theta$ 's truth. However, perhaps some predicates, the "observational" ones, should not be ramsified. This is the standard approach, associated with Carnap, Maxwell, Worrall and Zahar. Does Newman's objection apply here? The first discussion of this scenario was given in an interesting and very important 1985 article by William Demopoulos and Michael Friedman, who summarize the application of Newman's objection in this case as follows:

[I]f our theory is consistent, and if all its purely observational consequences are true, then the truth of the Ramsey-sentence follows as a theorem of set theory or second-order logic, provided our initial domain has the right cardinality - if it doesn't, then the consistency of our theory again implies the existence of a domain that does. (Demopoulos \& Friedman 1985, p.635.) 
The implications seem to be devastating:

(i) Epistemological structuralism reduces to a version of anti-realism (constructive empiricism, instrumentalism, or something similar).

(ii) Ontological structuralism reduces to the claim that every scientific theory can be re-expressed as "the world has cardinality at least $\mathrm{\kappa}$ ".

The main technical conclusion given by Demopoulos and Friedman - let us call it $D F$ - has been endorsed by several authors in the recent literature on the scientific realism debate (e.g., Psillos 1999). My intention in Ketland 2004 was to clarify the matter by providing an appropriate framework for formalizing scientific theories and seeing how to prove $D F$. As it turns out, $D F$ formulated as above is not quite correct: the main block in the proof is a kind of $\omega$-inconsistency (see footnote 10 below for some details). However, with certain modifications to the formulation of $D F$, one gets closely connected results (see, e.g., Theorems 1,4 and 5 below). The main result of Ketland 2004, using a 2-sorted formalization of the language of scientific theories, was:

$\Re(\Theta)$ is true iff $\Theta$ has a full model which is T-cardinality correct and empirically correct.

Thus, ramsification threatens to trivialize the content of scientific knowledge. For structural claims are, in effect, cardinality claims. Recently, there has been much further discussion of the Newman-DemopoulosFriedman objection. The two most interesting discussions, in my view, are Cruse 2005 and Melia \& Saatsi 2006. Melia and Saatsi propose to introduce into theory formulations certain higher-order modal relations between theoretical relations. I am not convinced that this approach will work. Cruse's points, concerning the observation-theory distinction, are more closely relevant to what is discussed below. ${ }^{6}$

\footnotetext{
${ }^{6}$ See Ainsworth 2009 for a clear survey of the main results, and some new ones, and an excellent discussion of some of the main lines of reply that have been given to Newman's objection.
} 


\section{FormaLIZATION OF SCIENTIFIC THEORIES}

\subsection{Empirical Adequacy and the O/T Distinction}

Below, we introduce two formalization schemes for scientific theories. The notion of "empirical adequacy" has to be defined somehow. Trying to do this carefully, one notices two notions of observationality at play. First, the notion of observable objects; second, the notion of observational predicates (or relations). In light of this, one might argue that an observational predicate could, in principle, be true of unobservable objects (or could relate observable objects to unobservable ones, or even unobservable objects to unobservable ones). Consider the predicate "part of". Suppose we take "part-of" to be observational. Consider the following three statements:

(23a) My left thumb is a part of my left hand.

(23b) There are blood cells which are parts of my left thumb.

(23c) There are molecules which are parts of blood cells.

Statement (23a) involves a relation between observable objects; an observable state of affairs. Statement (23b) is mixed: the corresponding state of affairs involves a relation holding between an unobservable objects and an observable one. Statement (23c) concerns a relation holding between unobservable objects. So, does "part of" count as an observational predicate? Must an "observational" predicate apply only to observable objects?

If we say $\mathrm{No}$, then the obvious 1-sorted way to formalize scientific theories leads to "observational" statements whose truth value depends upon the properties of unobservable objects. If we say Yes, then one cannot get a theorem of the kind that Demopoulos and Friedman were after unless one uses the 2-sorted formalization used in Ketland 2004. On the 2-sorted approach, we represent (23a)-(23c) as involving three distinct predicates, say "part ${ }_{\mathrm{O}}$ ", "part ${ }_{\mathrm{M}}$ " and "part $\mathrm{T}$ ", depending on the observational status of their relata. So, the empiricist desire to impose an $\mathrm{O} / \mathrm{T}$ distinction has forced us to "split" the part-of relation into three separate relations. ${ }^{7}$ How-

\footnotetext{
${ }^{7}$ I described this "relation-splitting" objection to the O/T distinction in talks in 2002 (at LSE and Leeds); in Ketland 2004, I commented: "The technical framework described here ... is riddled with further problems ... [M] any scientifically significant relations and quantities will 'decompose' into three strangely distinct relations, depending upon the observational status of their relata" (Ketland 2004, p.289, footnotes 3 and 4.)
} 
ever, on this 2-sorted approach, the observational predicates do only have observable objects as their relata; and one then obtains a correct $D F$-style result.

A less metaphysically artificial approach introduces either a distinction in the semantics (by supposing that some special subset $O b s$ of the domain $W$ of the intended interpretation consists of the observable objects) or by explicitly introducing into the theory-formulation language a primitive unary predicate $\operatorname{Obs}(x)$ meaning " $x$ is an observable object", and defining the observationla statements by restricting quantifiers to $O b s(x)$. (Cf., in set theory, one can restrict quantifiers to a given set, e.g., $\omega$.) This is, in effect, a 1-sorted formalization, in which the observable objects are explicitly distinguished by some predicate. We shall briefly discuss this approach below, in Section 3. However, one cannot then get a $D F$-style result, because the relevant notion of "empirical adequacy" is too weak to entail the truth of the Ramsey sentence (see the remarks after Definition E below).

However, the usual approach, associated with Carnap, is to consider a 1 -sorted theory-formulation language $L$ with primitive predicates partitioned into "observational" and "theoretical" ones, saying nothing about the observational status of the objects to which these predicates apply. In particular, the domain $W$ of the interpreted language may contain unobservables and the O-predicates are permitted to apply to these. On this approach, one may define a notion of "O-adequacy" and one obtains a $D F$ style result, " $\mathfrak{R}(\Theta)$ is true iff $\Theta$ is $\mathrm{O}$-adequate". However, the problem is that "O-adequate" doesn't mean what is intended. For the quantifiers of $L$ range over all elements of $W$, including unobservables. So, certain "observational" statements will be true/false in virtue of facts about unobservable objects.

\subsection{One-Sorted Formalization}

Scientific theories are to be formulated in a one-sorted, second-order, theory formulation language $L$ with identity. Non-logical primitives of $L$ are classified into two kinds: observational predicates, $\mathbf{O}_{i}$, and theoretical predicates, $\mathbf{T}_{i}$. The sublanguage of $L$ from which the theoretical predicates and second-order variables have been deleted is called L's observational sublanguage, denoted $L_{0}$. An $L$-theory $\Theta$ is a deductively closed set of $L$ sentences. We may identify a theory $\Theta$ with some axiomatization of it; if $\Theta$ is finitely axiomatizable, then we may identify $\Theta$ with a single formula which is the conjunction of the axioms. Suppose $\Theta$ is the single axiom for 
a finitely axiomatized $L$-theory. The Ramsey sentence $\Re(\Theta)$ is obtained by replacing, in $\Theta$, each occurrence of a theoretical predicate $\mathbf{T}_{i}$ by a secondorder variable $X_{i}$ of the same arity, and prefixing the result with a string of existential quantifiers, one for each variable $X_{i}$ that appears. Thus,

\section{Definition A}

$\mathfrak{R}(\Theta)$ is: $\exists X_{1} \ldots \exists X_{n} \Theta\left(\mathbf{T}_{1} / X_{1}, \ldots, \mathbf{T}_{n} / X_{n}\right)$.

$L$ is given a special partial interpretation $\left(W,\left\{O_{i}\right\}\right)$, where $W$ is the domain over which the quantifiers range and the $O_{i}$ are the "observational" relations on $W$ which interpret the observational predicates $\mathbf{O}_{i}$. We suppose that $W=O b s \cup U n o b s$, where $O b s$ is the set of "observable" objects and Unobs is the set of "unobservable" objects. (Obs and Unobs are assumed disjoint.) As discussed above, we allow "observational" predicates to apply to some unobservable objects. The semantics is standard Tarskian semantics. $^{8}$

\subsection{Two-Sorted Formalization}

The language $L$ is now a two-sorted, second-order, language with identity. The non-logical primitives of $L$ are classified into three kinds: observational, $\mathbf{O}_{i}$; mixed, $\mathbf{M}_{i}$; and theoretical, $\mathbf{T}_{i}$. The sublanguage from which the theoretical and mixed predicates have been deleted is $L$ 's observational sublanguage, denoted $L_{\mathrm{O}}$. If $\Theta$ is a finitely axiomatized theory in $L$, the Ramsey sentence $\mathfrak{R}(\Theta)$ is obtained by ramsifying the theoretical and mixed predicates. $L$ has a special 2-sorted partial interpretation $\left(\left(D_{\mathrm{O}}, D_{\mathrm{T}}\right),\left\{O_{i}\right\}\right)$, where $D_{\mathrm{O}}$ is the domain of observable objects and $D_{\mathrm{T}}$ is the domain of unobservable objects. (These are disjoint.) The two sorts of variable range over these distinct domains.

\footnotetext{
${ }^{8}$ For second-order sentences of $L$, standard full semantics is assumed.
} 
As noted above, the relevant notion of empirical adequacy is tricky to formulate on the 1-sorted formalization.

\section{Definition B}

The substructure $\left(O b s,\left\{\left.O_{i}\right|_{O b s}\right\}\right)$, generated by the subset $O b s$, is the empirical substructure of $\left(W,\left\{O_{i}\right\}\right)$.

\section{Definition C}

An $L$-theory $\Theta$ is weakly empirically adequate iff all $L_{\mathrm{O}}$-theorems of $\Theta$ are true in $\left(O b s,\left\{\left.O_{i}\right|_{\text {obs }}\right\}\right)$.

Perhaps a better analysis of the notion of empirical adequacy requires an embedding of the empirical substructure into some model of $\Theta$. That is:

\section{Definition D}

An $L$-structure $\mathbf{M}=\left(D,\left\{R_{i}^{O}\right\},\left\{R_{i}^{T}\right\}\right)$ is empirically correct iff there is an embedding $f:\left(O b s,\left\{\left.O_{i}\right|_{O b s}\right\}\right) \rightarrow\left(D,\left\{R_{i}^{O}\right\}\right)$.

\section{Definition E}

An $L$-theory $\Theta$ is empirically adequate iff $\Theta$ has a full model which is empirically correct.

Sadly, Definitions A-E are pointless. For the adequacy conditions on $\Theta$ given in Definitions $\mathrm{C}$ and $\mathrm{E}$ are too weak to imply the truth of $\Re(\Theta)$. As it turns out, if we want $D F$-style results, we must either move to the 2-sorted formalization or introduce a stronger notion, "O-adequacy":

\section{Definition F}

An $L$-theory $\Theta$ is weakly $\mathrm{O}$-adequate iff all $L_{\mathrm{O}}$-theorems of $\Theta$ are true.

\section{Definition G}

An $L$-structure $\mathbf{M}=\left(D,\left\{R_{i}^{\mathrm{O}}\right\},\left\{R_{i}^{\mathrm{T}}\right\}\right)$ is O-correct iff $\left(D,\left\{R_{i}^{\mathrm{O}}\right\}\right) \cong$ $\left(W,\left\{O_{i}\right\}\right)$.

\section{Definition $\mathbf{H}$}

An $L$-theory $\Theta$ is O-adequate iff $\Theta$ has a full, O-correct, model. 
Next, assume the 2-sorted formalization.

\section{Definition I}

A 2-sorted $L$-structure $\mathbf{M}=\left(\left(D_{1}, D_{2}\right),\left\{R_{i}^{\mathrm{O}}\right\},\left\{R_{i}^{\mathrm{M}}\right\},\left\{R_{i}^{\mathrm{T}}\right\}\right)$ is empirically correct iff $\left(D_{1},\left\{R_{i}{ }^{\mathrm{O}}\right\}\right) \cong\left(D_{\mathrm{O}},\left\{O_{i}\right\}\right)$.

\section{Definition J}

A 2-sorted $L$-structure $\mathbf{M}=\left(\left(D_{1}, D_{2}\right),\left\{R_{i}^{\mathrm{O}}\right\},\left\{R_{i}^{\mathrm{M}}\right\},\left\{R_{i}^{\mathrm{T}}\right\}\right)$ is T-cardinality correct iff there is a bijection $f: D_{2} \rightarrow D_{\mathrm{T}}$.

\section{MAIN RESUlts}

On the 1-sorted formalization, we first show that $\mathfrak{R}(\Theta)$ is true iff $\left(W,\left\{O_{i}\right\}\right)$ can be expanded to a full model of $\Theta$. Hence:

Theorem 1: $\mathfrak{R}(\Theta)$ is true iff $\Theta$ is $\mathrm{O}$-adequate.

Note that $\mathrm{O}$-adequacy (Definition $\mathrm{H}$ ) is stronger than empirical adequacy (Definition E). So, $\Re(\Theta)$ 's truth is not necessarily implied by $\Theta$ 's empirical adequacy. Next, consider whether weak $\mathrm{O}$-adequacy is sufficient for the truth of $\mathfrak{R}(\Theta)$. First, we can show,

Theorem 2: If $\Theta$ is $\mathrm{O}$-adequate, then $\Theta$ is weakly $\mathrm{O}$-adequate.

However, the converse is not true:

\section{Theorem 3}

There are weakly $\mathrm{O}$-adequate theories $\Theta$ which are not $\mathrm{O}$-adequate.

This occurs only when the intended domain $W$ is infinite. We can fix things up a bit:

Theorem 4: Suppose $W$ is finite. Then $\Re(\Theta)$ is true iff $\Theta$ is weakly O-adequate. ${ }^{9}$

\footnotetext{
${ }^{9}$ The proof uses the following lemma: Let $L^{*}$ be a sublanguage of $L$. Suppose $\Theta^{*}$ is the set of $L^{*}$-theorems of $\Theta$. Then any finite model of $\Theta^{*}$ can be expanded to a model of $\Theta$.
} 
This is the closest we get, on the 1-sorted formalization, to the original claim of Demopoulos \& Friedman 1985, which spoke of the truth of "all purely observational consequences" (i.e., weak adequacy). Two comments are worth making. First, we must use the notion of "weak O-adequacy" rather than the notion of weak empirical adequacy; second, the domain $W$ of the intended interpretation must be finite. ${ }^{10}$

On the 2-sorted formalization, we obtain,

\section{Theorem 5}

$\mathfrak{R}(\Theta)$ is true iff $\Theta$ has a full empirically correct and T-cardinality correct model.

In this framework, the truth condition for $\mathfrak{R}(\Theta)$ now involves empirical adequacy, not O-adequacy: this was the motivation for using this framework in Ketland 2004. However, Cruse 2005 has criticized my decision to ramsify the "mixed" predicates on the 2-sorted formalization. Cruse argues that mixed predicates should count as "observational" and thus shouldn't be ramsified. However, this definition of "observational predicate" becomes analogous to the notion of O-predicate on the 1-sorted approach. One can then give a definition of an "O-M correct" structure:

\section{Definition K}

An $L$-structure $\left(\left(D_{1}, D_{2}\right),\left\{R_{i}^{\mathrm{O}}\right\},\left\{R_{i}^{\mathrm{M}}\right\},\left\{R_{i}^{\mathrm{T}}\right\}\right)$ is O-M correct iff $\left(\left(D_{1}, D_{2}\right),\left\{R_{i}^{\mathrm{O}}\right\},\left\{R_{i}^{\mathrm{M}}\right\}\right) \cong\left(\left(D_{\mathrm{O}}, D_{\mathrm{T}}\right),\left\{O_{\mathrm{i}}\right\},\left\{M_{i}\right\}\right)$.

A modification of the proof of Theorem 5 gives:

Theorem 6: $\mathfrak{R}(\Theta)$ is true iff $\Theta$ has a full model which is $\mathrm{O}-\mathrm{M}$ correct.

\footnotetext{
${ }^{10}$ If we admit possible worlds containing an infinity of observables, resembling the natural numbers, then counterexamples to the original $D F$ claim can be given. In Ketland 2004, one is given based on the $\omega$-inconsistent Friedman-Sheard truth theory over Peano arithmetic, using results obtained by McGee 1985 and Halbach 1999. A similar counterexample can be given based on the $\omega$-inconsistent, conservative extension of Peano arithmetic, based on Yablo's paradox, discussed in Ketland 2005. Another counterexample appeared in van Benthem 1978, based on the (r.e.) theory of $(\omega,<)$ extended by axioms saying "there is a T", but " $0 \notin \mathrm{T}$ and, for all $x$, if $x \notin \mathrm{T}, s(x) \notin \mathrm{T}$ ". In each case, we obtain examples of theories which are weakly $\mathrm{O}$-adequate (and have a model of the right size), but their Ramsey sentences are not true.
} 
This is Theorem 1 in disguise. $\Re(\Theta)$ 's truth condition now involves facts about unobservables. However, information about the purely theoretical relationships amongst unobservables has again been reduced to mere cardinality (encoded into the bijections required for O-M correctness). So, this response achieves little.

\section{CONCLUding COMMENTS}

It seems to me that the results above confirm Demopoulos and Friedman's main conclusion that ramsification "trivializes physics" (Demopoulos \& Friedman 1985, p.635); ramsification leads to what might be called physics without physics. That is, purely structural content just is cardinality content. ${ }^{11}$ So far as I can see, if one wishes to avoid such conclusions, one must adopt a form of scientific essentialism or natural kinds realism:

\section{Scientific Essentialism/Natural Kinds Realism}

Nature has its own special "natural kind" structure; second-order variables only range over the relations that are "natural".

Thus, Ramsey sentences should be understood as follows:

(24) There are natural relations $R_{1}, \ldots, R_{n}$ such that $\Theta\left(R_{1}, \ldots, R_{n}\right)$.

However, is scientific essentialism consistent with scientific structuralism? The structuralist, epistemological or ontological, must take the metaphysical notion of "natural relation" as an unreduced primitive in theorizing, and must assume from the outset that reality has a built-in natural kind structure.

\section{REFERENCES}

Ainsworth, P. 2009: "Newman's Objection". B.J.P.S. 60, pp.135-171.

Brading, K. and E. Landry. 2005: "Scientific Structuralism: Presentation and Representation". Philosophy of Science 73, pp.571-581.

${ }^{11}$ Roy Cook put it as follows: ramsification "reduces physics to counting". 
Carnap, R. 1956: The Philosophical Foundations of Physics, edited by M. Gardner. New York: Basic Books. Reprinted as An Introduction to the Philosophy of Science, New York: Dover Books, 1995.

Cruse, P. 2005: "Ramsey Sentences, Structural Realism and Trivial Realization". Studies in the History and Philosophy of Science 36, pp.557-576.

Demopoulos, W and M. Friedman 1985: "Critical Notice: Bertrand Russell's The Analysis of Matter: Its Historical Context and Contemporary Interest", Philosophy of Science 52, pp.621-639.

Halbach, V. 1999: "Conservative Theories of Classical Truth". Studia Logica 62, pp.353-370.

Ketland, J. 2004: "Empirical Adequacy and Ramsification". B.J.P.S. 55, pp.287-300.

Ketland, J. 2005: "Yablo's Paradox and $\omega$-Inconsistency". Synthese 145, pp.295-302.

Ladyman, J. 1998. "What is Structural Realism?", Studies in the History and Philosophy of Science 29, pp.409-424.

Lewis, D. 1970: "How to Define Theoretical Terms". Journal of Philosophy 67, pp.427-446.

Maxwell, G. 1970. "Structural Realism and the Meaning of Theoretical Terms", in S. Winokur and M. Radner (eds), Analyses of Theories and Methods of Physics and Psychology, Minneapolis, MN: University of Minnesota Press, pp.181-192.

McGee, V. 1985: 'How Truthlike can a Predicate Be? A Negative Result', Journal of Philosophical Logic 14, pp.399-411.

Melia, J. and J. Saatsi. 2006: "Ramseyfication and Theoretical Content". B.J.P.S. 57, pp.561-585.

Newman, M.H.A. 1928: "Mr Russell's Causal Theory of Perception". Mind 37, pp.137-148.

Psillos, S. 1999: Scientific Realism: How Science Tracks Truth. London: Routledge.

Ramsey, F.P. 1929: “Theories”, in R.B. Braithwaite (ed.), 1931, Foundations of Mathematics, London: Routledge and Kegan Paul.

Russell, B.A.W. 1919. A Mathematical Introduction to Philosophy. London: Allen and Unwin.

Russell, B.A.W 1927: Analysis of Matter. London: Allen and Unwin.

van Benthem, J. 1978: "Ramsey Eliminability". Studia Logica 37, pp.321-336.

van Fraassen, B.C. 1980: The Scientific Image. Oxford: Clarendon Press.

Worrall, J. 1989: "Structural Realism: The Best of Both Worlds?". Dialectica 43, pp.99-124.

Zahar, E. 2001: Poincaré's Philosophy: From Conventionalism to Phenomenology. Chicago: Open Court. 
